\title{
An Evaluation of the Efficacy and Characterization of a Cross-Linked Hyaluronic Acid/Collagen/Poloxamer Sheet for Use in Chronic Wounds
}

\author{
Hwi Ju Kang, Yu Jin Jang, II Kyu Park, Hyun Jung Kim
}

R\&D Center, Genewel Co., Ltd., Seongnam, Korea

\begin{abstract}
Background: Numerous multidirectional studies have been conducted on the use of hyaluronic acid (HA) and collagen in the treatment of chronic wounds. A complex manufacturing process is involved in contemporary cross-linking methods. Thus, the current study objectives were to improve this process through the development of a cross-linked HA (C$\mathrm{HA}$ ), collagen, and poloxamer dressing material, and to evaluate its characteristics and efficacy in the treatment of chronic wounds.

Methods: C-HA, collagen, and poloxamer sheets were produced. C-HA gel was prepared and homogenized with collagen and poloxamer. Thereafter, it was aliquoted, followed by freeze drying. The surface of sheets was evaluated using scanning electron microscopy, and its tensile strength and properties were measured. The amount of residual crosslinking agent in the HA gel was measured using gas chromatography and the cytotoxicity of the sheets was evaluated according to the ISO10993 criteria. Wound healing was assessed in a type 1 diabetes mellitus model.

Results: The sheet was found to have a uniform, porous structure, with adequate strength and sufficient absorption to absorb the exudate. Less than $2 \mathrm{ppm}$ of residual cross-linking agent was detected and cytotoxicity was not observed, indicating the safety of the product. The wound healing rate in the type 1 diabetes mellitus model treated with the dressing was observed to be greater than that in the untreated group.

Conclusion: The use of a newly developed cross-linked HA/collagen/poloxamer sheet was demonstrated to facilitate wound healing in patients with chronic wounds owing to its structure and healing properties.
\end{abstract}

Keywords: Hyaluronic acid, Collagen, Poloxamer, Cross-linked, Chronic wound

\section{Introduction}

Various types of extracellular matrices (ECMs) in the skin and body play an important role in the formation of body parts, such as skin or muscle. Hyaluronic acid $(\mathrm{HA})$ is a major component of the ECM which is predominantly present in the skin, joints, soft tissues, and synovial fluid [1,2]. As it is abundant in the skin, HA is known to play an important biological role in the wound healing process in the skin [3]. It is also known to have excellent biocompatibility and non-immunogenicity.

Collagen is also present in most body tissues and contributes to the stability and structure of tissues and organs owing to its characteristic molecular structure. Collagen has excellent hygroscopicity and biocompatibility, and promotes cell migration, proliferation, and vascularization [4].

Thus, HA and collagen, considered to be safe materials in the body, have been used in a wide variety of applications, such as wound dressings, tissue regeneration, and tissue filling $[5,6]$. When these materials are combined for use in tissue regeneration and tissue filling, in particular, diverse cross-linking methods are employed

\section{Original Article}

Received: February 12, 2018

Accepted: March 16, 2018

\section{Corresponding author: \\ Hwi Ju Kang, M.S.}

Genewel, 6F, Star Tower, 37 Sagimakgol-ro 62beon-gil, Jungwon-gu, Seongnam 13211, Korea

Tel: +82-31-8018-5500

Fax: +82-31-8018-5509

E-mail: aneliss@idongsung.com

This is an Open Access article distributed under the terms of the Creative Commons Attribution Non-Commercial License (http://creativecommons.org/licenses/by-nc/4.0/) which permits unrestricted non-commercial use, distribution, and reproduction in any medium, provided the original work is properly cited.

C 2018 Korean Wound Management Society 
because they need to be maintained in the body for a certain period.

The 1-ethyl-3-(3-dimethylaminopropyl) carbodiimide (EDC) and 1,4-butanediol diglycidyl ether (BDDE) cross-linking methods are the most commonly used $[7,8]$. To manufacture tissue engineering support or a wound dressing, $\mathrm{HA}$ and collagen are cross-linked using a specific agent for this purpose and are then subjected to an freeze-drying process using the desired mold shape. A washing process removes the residual toxicity of the cross-linking agent. The HA/collagen sheet or scaffold is usually obtained after a second freeze-drying process is carried out. However, since this is performed over at least two days, currently many restrictions are imposed on the manufacturing time in the manufacture of a large amount of support or dressing.

In an effort to simplify the two-step freeze-drying process, the current study objective was to develop C-HA sheets by mixing the first freeze-dried product with collagen. BDDE-C$\mathrm{HA}$ gel was used to prepare the C-HA/collagen/poloxamer sheets. Analysis of the characteristics of the product was performed to select the biocompatible sheet most suited to manufacturing. A type 1 diabetes mellitus model, widely used in the treatment of chronic wounds, was employed. The wound healing efficacy of the finally selected sheet was also assessed.

\section{Methods}

\section{Materials}

Sodium hyaluronate (HA) was purchased from Bloomage (China). BDDE and sodium hydroxide $(\mathrm{NaOH})$ were purchased from Sigma Aldrich (USA). To prepare phosphatebuffered saline (PBS) with a $\mathrm{pH}$ of 7.4, sodium chloride, sodium phosphate dibasic and potassium phosphate monobasic were obtained from Daejung Chemicals (Korea). Poloxamer (Kolliphor ${ }^{\circledR} \mathrm{P}$ 188) was purchased from BASF (USAP) and atelocollagen was extracted from pigs before use.

\section{Preparation of cross linked-hyaluronic acid}

Sodium hyaluronate (HA) was dissolved in $0.25 \mathrm{~N} \mathrm{NaOH}$ solution to make a $10 \%$ solution, to which BDDE was added, in a BDDE/HA molar ratio of $0.05,0.07,0.09$, and 0.11 . The solution was incubated overnight at room temperature. It was then washed eight times with PBS hourly and once with purified water. The purified C-HA was pulverized and stored in the refrigerator once its solid content had been measured.

\section{Measurement of BDDE residual}

C-HA was then eluted in distilled water at $0.2 \mathrm{~g} / \mathrm{mL}$ for 72 hours. To this solution, equal volumes of $10 \mathrm{ppm}$ of dipropyl phthalate dissolved in ethyl acetate were added, and the resulting solution was subjected to voltexing for an hour, followed by centrifugation at 1,500 rpm for five minutes. Following centrifugation, the supernatant was collected and used as the test solution. To prepare the reference solution, BDDE was dissolved in saline solution at 2 ppm, as per the safety recommendations of US Food and Drug Administration.

The reference and test solutions were analyzed using gas chromatography (GC) (Agilent, USA). HP-5 (Agilent) was used as a column, with a flow rate of $0.5 \mathrm{~mL} / \mathrm{min}$ in the temperature range of $150-250^{\circ} \mathrm{C}$. A flame ionization detector was used as a detector.

\section{Viscosity of cross linked-hyaluronic acid}

Distilled water was added to ensure C-HA with a solid content of $1 \%$. The viscosity of the prepared $1 \% \mathrm{C}-\mathrm{HA}$ was then measured using a viscometer (Brookfield, USA).

\section{Preparation of $\mathrm{C}-\mathrm{HA} /$ collagen/poloxamer sheet}

The sheet was created using the C-HA prepared with $2 \%$ solid content. Atelocollagen (0.1 g) was added to $59.8 \mathrm{~g}$ of the distilled water (with a pH adjusted to below 3) and the solution was completely dissolved using a homogenizer. The $\mathrm{pH}$ was further adjusted to 7 with the addition of $\mathrm{NaOH}$. Thereafter, $40 \mathrm{~g}$ of $\mathrm{C}-\mathrm{HA}$ was added and mixed with a homogenizer for 15 minutes, to which $0.1 \mathrm{~g}$ of poloxamer was added and mixed once more using a homogenizer for a further 30 minutes. The prepared solution was dispensed into a 12.5 $\mathrm{cm} \times 12.5 \mathrm{~cm}$ square dish and freeze dried to obtain a $3 \mathrm{~mm}$ thick sheet. Preparation of the C-HA/collagen/poloxamer sheet is depicted in Fig. 1.

\section{Characterization of $\mathrm{C}-\mathrm{HA} /$ collagen/poloxamer sheet Morphology \\ The C-HA/collagen/poloxamer sheet was cut and its surface was coated with Au-Pd. Thereafter, the surface structure was analyzed using scanning electron microscope (SEM; CX- $100 \times$, Coxem, Korea).}

Tensile strength

The sheets were cut into pieces in the shape of a dog-bone, some of which were placed in water for 30 minutes for a comparison of tensile strength under wet conditions. Tensile 

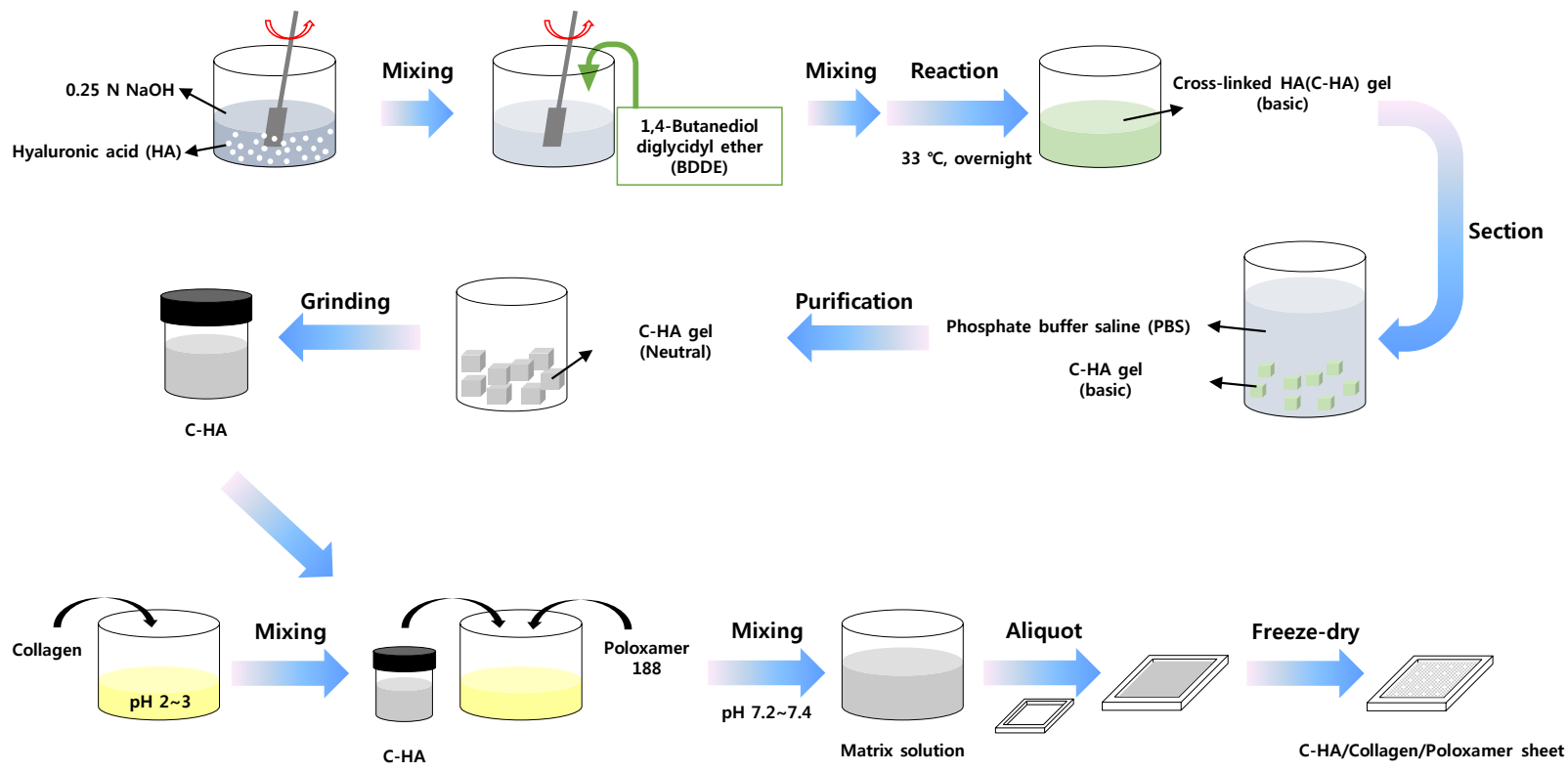

Fig. 1. Preparation of $\mathrm{C}-\mathrm{HA} /$ collagen/poloxamer sheet.

testing was performed using an Universal Testing Machine (3343, Instron, USA) at a cross-head speed of $12.5 \mathrm{~mm}$ to measure the maximum stress values.

\section{Water absorption}

Four different C-HA/collagen/poloxamer sheets, prepared according to various cross-linking degrees, were cut into $3 \mathrm{~cm}$ $\times 3 \mathrm{~cm}$ pieces, weighed, placed in water, and then weighed once more after 30 minutes. The degree of swelling was calculated by dividing the difference between the weight (Ws) of each swelled and dried sheet (Wd) by the weight of the dried sheet, and then expressing it as a percentage:

$$
\text { Water absorption }(\%)=\frac{(W s-W d)}{W s} \times 100
$$

\section{Disintegration test of $\mathrm{C}-\mathrm{HA} /$ collagen/poloxamer sheet}

The sheets were then cut into $2 \times 2 \mathrm{~cm}$ pieces and their initial weight (WO) was measured. Following the addition of $10 \mathrm{~mL}$ of PBS, the cut sheets were stirred at $50 \mathrm{rpm}$ for seven days at $32^{\circ} \mathrm{C}$ to determine the extent of disintegration. They were then washed with distilled water and dried. Their dried weight $(\mathrm{Wt})$ was measured to determine the extent of disintegration based on the weight loss:

$$
\text { Weight loss }(\%)=\frac{(W 0-W t)}{W 0} \times 100
$$

\section{Cytotoxicity}

A cell counting method was used to assess the toxicity of the product. L929 cells (CCL-1, ATCC, USA) were seeded in a 6 -well plate $\left(2 \times 10^{5}\right.$ cells/well) and cultured for 24 hours. Four sheets, with different degrees of cross-linking, were sterilized with ethylene oxide. Each of them was cut into a 60 $\mathrm{cm}^{2}$ piece and added to $20 \mathrm{~mL}$ of the cell culture medium to incubate at $37^{\circ} \mathrm{C}$ for elution. Two $\mathrm{mL}$ of eluate was added to each well of the 6-well plate containing cells and the cells were cultured for a further 24 hours. The elute was then removed. The cells were washed with PBS and trypsinized, and the number of cells per well was counted

\section{In vivo wound healing experiment}

Preparation of type I diabetic mouse model

ICR mice (male, aged seven weeks) were purchased from Orient Bio and included in the study after a week of acclimatization. Streptozocin (STZ; Sigma-Aldrich) was dissolved in $0.05 \mathrm{M}$ citrate buffer ( $\mathrm{pH}$ of 4.5 ) and administered to the mice by intraperitoneal injection at $200 \mathrm{mg} / \mathrm{kg}$ body weight to induce type I diabetes mellitus. After a week, the blood glucose levels of the mice were confirmed to be $\geq 300 \mathrm{mg} / \mathrm{dL}$.

Ethics approval and consent to participate

The experiment and management of laboratory animals were conducted according to relevant guidelines after review and approved by Institutional Animal Care and Use Committee 

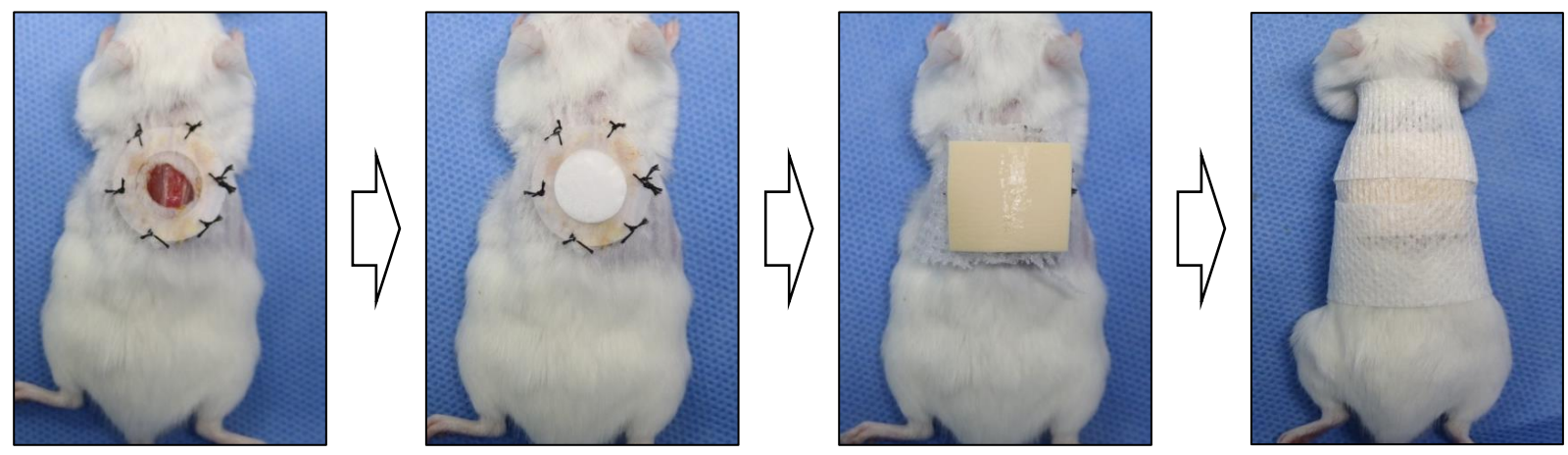

Fig. 2. The surgical procedure followed. A wound of $10 \mathrm{~mm}$ in diameter was induced and fixed with a silicone ring. After the sheet was treated, it was covered with a secondary dressing and then taped.

approved (Approval number: GAP-AVAL-17012).

Experimental wound model

The wound healing model was prepared by epilating the whole back area of the mice and then making a full-thickness wound of $10 \mathrm{~mm}$ in diameter at the center of the back. A silicone ring was fixed with a suture to fix the wound. The sheets were cut to the size of the wound and sterilized with ethylene oxide prior to use. A sterilized sheet was placed on each wound and dressed to protect it from the external environment. The mice were placed under a warming lamp to monitor their recovery after anesthesia. Details of the surgical procedure followed are provided in Fig. 2.

The mice that were not treated with the sheet were used as the control group, and the rodents to whom the C-HA/collagen/poloxamer sheet (prepared with a BDDE/HA molar ratio of 0.11) was applied constituted the experimental group. The dressing was replaced on days 3,7 , and 10 following treatment. Three mice per group were sacrificed for histologic analysis on days 7 and 14 .

\section{Histological analysis}

Thereafter, the wound tissue was collected and fixed in 10\% formalin solution. The fixed tissue was embedded in paraffin through pretreatment. The tissue specimens were prepared to a thickness of $5 \mu \mathrm{m}$ and were subjected to hematoxylin and eosin (H\&E) and Masson's trichrome (MT) staining. The stained tissue specimens were analyzed for wound re-epithelialization and collagen deposition using Image J software.

\section{Statistical analysis}

A statistical analysis was performed using the Student's t-test. Statistical significance was set at a P-value of $<0.05$.
Table 1. Measurements of the residual amount BDDE

\begin{tabular}{lllll}
\hline BDDE/HA molar ratio & 0.05 & 0.07 & 0.09 & 0.11 \\
BDDE concentration (ppm) & 0.73 & 0.92 & 0.46 & 0.27 \\
\hline
\end{tabular}

\section{Results}

\section{Measurement of BDDE residual}

The amount of residual BDDE in the prepared $\mathrm{C}-\mathrm{HA}$ gel was measured using GC to determine the safety properties of the final product (Table 1 ). It was found to be $\leq 1 \mathrm{ppm}$, regardless of the BDDE/HA molar ratio, i.e., below the acceptable limit of 2 ppm for medical devices. Therefore, it was concluded to be safe.

\section{Viscosity}

It was found that the surface of the sheets produced by this study was rugged under certain conditions. Viscosity of the C-HA solution, rather than the freeze-drying process, were confirmed to be related to the surface of the sheet. Viscosity of less than 1,000 cP was recorded for sheets made with a $\mathrm{BDDE} / \mathrm{HA}$ molar ratio of $\leq 0.11$. When other sheets were created for comparison, it was found that those with a uniform surface were produced when viscosity of $\mathrm{C}-\mathrm{HA}$ was $1,000 \mathrm{cP}$ or lower (Table 2).

\section{Morphology}

The surface morphology of the C-HA/collagen/poloxamer sheets, made using $\mathrm{C}-\mathrm{HA}$ prepared with a BDDE/HA molar ratio of 0.05-0.11, was confirmed using SEM (Fig. 3). Pores of about $100 \mu \mathrm{m}$ were observed in the surface of the sheets. Pore size was seen to be similar, regardless of the BDDE/HA molar ratio. 

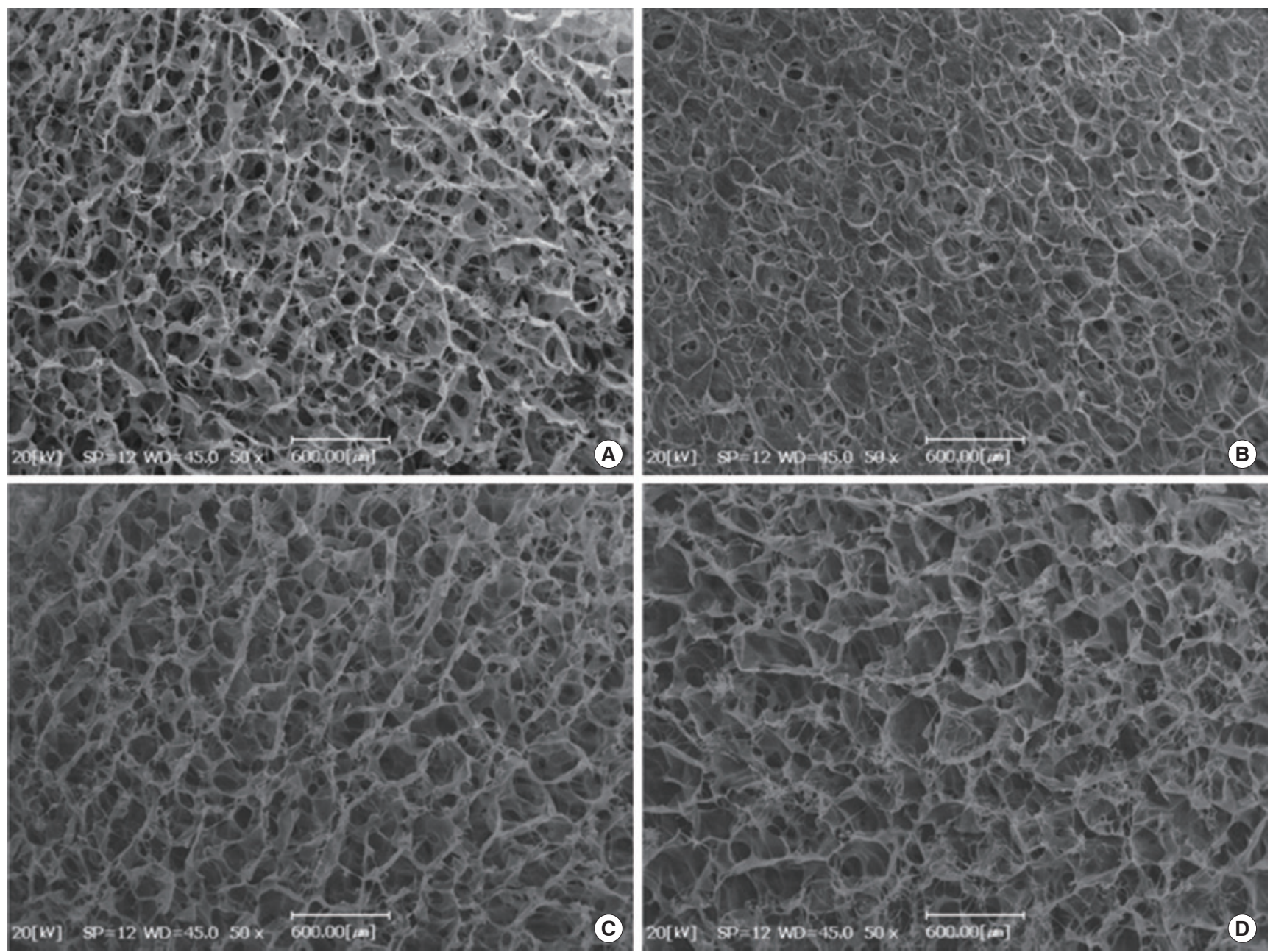

Fig. 3. Surface structure of C-HA/collagen/poloxamer sheet by SEM BDDE/HA molar ratio is (A) 0.05, (B) 0.07 , (C) 0.09 , and (D) 0.11 .

Table 2. Viscosity of $\mathrm{C}-\mathrm{HA}$

\begin{tabular}{lcccc}
\hline BDDE/HA & 0.05 & 0.07 & 0.09 & 0.11 \\
Viscosity of C-HA (cP) & $500 \pm 20$ & $560 \pm 20$ & $450 \pm 60$ & $920 \pm 130$ \\
\hline
\end{tabular}

Table 3. Tensile strength of the C-HA/collagen/poloxamer sheets according to the BDDE/HA molar ratio

\begin{tabular}{lcccc}
\hline BDDE/HA & 0.05 & 0.07 & 0.09 & 0.11 \\
Tensile strength $\left(\mathrm{N} / \mathrm{mm}^{2}\right)$ & $0.10 \pm 0.02$ & $0.10 \pm 0.02$ & $0.10 \pm 0.01$ & $0.30 \pm 0.03$
\end{tabular}

Tensile strength

Measurements were taken of the sheets prepared using the same $\mathrm{C}-\mathrm{HA}$ ratio and BDDE/HA molar ratio to determine the effect of this on the tensile strength of the sheets. The tensile strength was found to be similar at around $0.1 \mathrm{~N} / \mathrm{mm}^{2}$, regardless of changes to either the BDDE/HA molar ratios or $\mathrm{C}-\mathrm{HA}$ ratios (Tables 3,4 ). When the effect of the BDDE/HA
Table 4. Tensile strength of the C-HA/collagen/poloxamer sheets according to the $\mathrm{C}-\mathrm{HA}$ ratio

\begin{tabular}{lccc}
\hline C-HA/Collagen/P188 & $6 / 2 / 2$ & $7 / 1.5 .1 .5$ & $8 / 1 / 1$ \\
Tensile strength (N/mm $)$ & $0.07 \pm 0.01$ & $0.10 \pm 0.02$ & $0.10 \pm 0.01$ \\
\hline
\end{tabular}

Table 5. Tensile of the C-HA/collagen/poloxamer sheets according to the BDDE/HA molar ratio after wetting

\begin{tabular}{lcccc}
\hline BDDE/HA & 0.05 & 0.07 & 0.09 & 0.11 \\
$\begin{array}{c}\text { Tensile strength } \\
\left(\mathrm{N} / \mathrm{mm}^{2}\right)\end{array}$ & $0.011 \pm 0.001$ & $0.012 \pm 0.003$ & $0.011 \pm 0.003$ & $0.011 \pm 0.003$ \\
\hline
\end{tabular}

molar ratio on the strength of the sheets was assessed under wet conditions, the tensile strength was measured at an average of $0.011 \mathrm{~N} / \mathrm{mm}^{2}$ regardless of the BDDE/HA molar ratio, which was similar before wetting (Table 5). It was concluded that the degree of cross-linking did not affect the physical properties of the prepared sheets. 

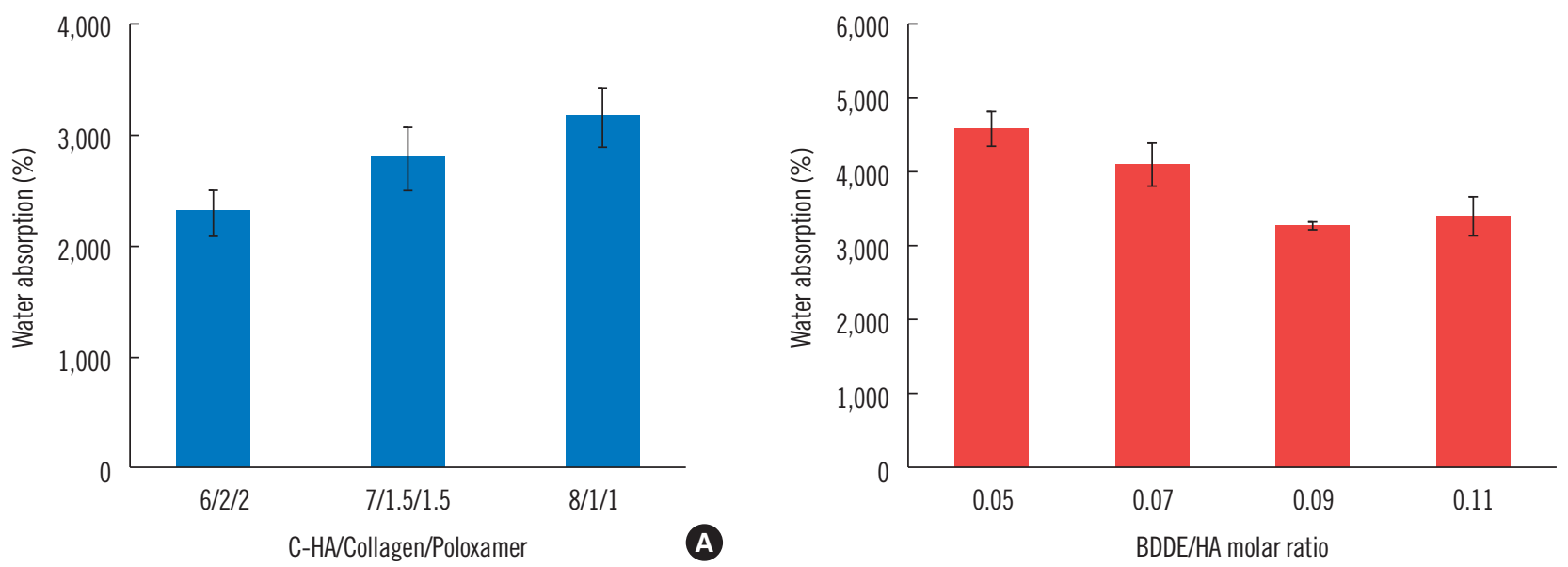

Fig. 4. Water absorption results based on (A) C-HA ratio and (B) BDDE/HA molar ratio.
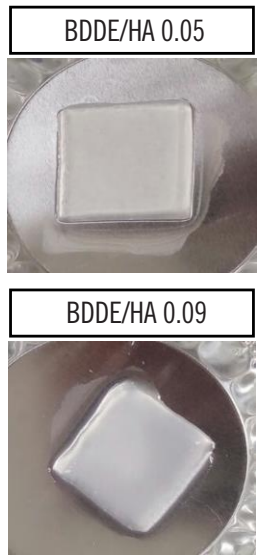
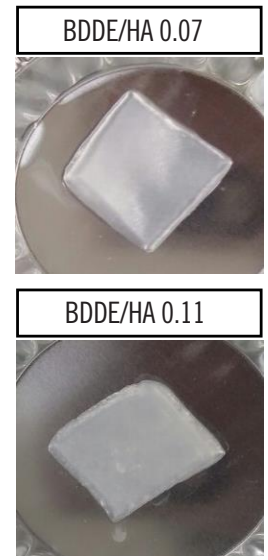

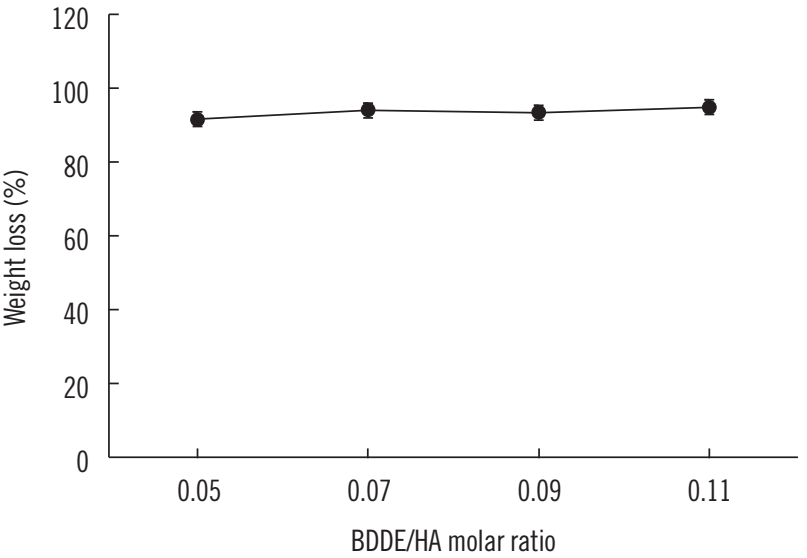

Fig. 5. (A) Appearance and (B) weight loss (\%) of the sheets after 7 days in PBS.

\section{Water absorption}

Measurements were taken of sheets prepared using the same $\mathrm{C}-\mathrm{HA}$ ratio and BDDE/HA molar ratio to determine the effect of this on absorption of the sheets. It was found that when the same BDDE/HA molar ratio was used, the higher the amount of $\mathrm{C}-\mathrm{HA}$, the greater the absorption. This was attributed to the absorption nature of HA. When the effect of the BDDE/HA molar ratio on absorption was examined, it was found that the smaller the BDDE/HA molar ratio, the greater the amount of absorption. However, a significant difference was not observed when using ratios from 0.09-0.11 (Fig. 4).

Disintegration test

A study objective was to evaluate the impact of the $\mathrm{C}-\mathrm{HA} / \mathrm{col}-$ lagen/poloxamer sheet on chronic wound healing in diabetic rodents. Therefore, an evaluation was performed of whether or not the sheets could maintain their shape without disinte- grating until replacement of the wound dressing. The extent to which disintegration occurred was determined by changes in the appearance and weight of the product after seven days in a PBS environment.

Regardless of the BDDE/HA molar ratio, the appearance of the sheet remained unaltered although the some weight changed. It seemed that mostly attributed to its physical entanglement between molecular chains during the pulverization process of the $\mathrm{C}-\mathrm{HA} /$ collagen (Fig. 5).

\section{Cytotoxicity}

Cytotoxicity testing is usually performed to determine biological safety and is the simplest in vitro experiment to evaluate. Increasingly, cell counting is regarded as a more reliable cytotoxicity testing method than the MTT method. Thus, cell counting, in conjunction with ISO 10993-5 compliant cytotoxicity tests, was employed to assess cytotoxicity in the current 
research. This took place after the elution step was applied to the $\mathrm{C}-\mathrm{HA} /$ collagen/poloxamer sheets, in accordance with the ISO 10993-12 requirements for medical devices.

Cell viability of $80 \%$ or higher was observed for all the sheets, regardless of the BDDE/HA molar ratio, thus indicating a lack of cytotoxicity (Fig. 6).
Wound healing effect of $\mathrm{C}-\mathrm{HA} /$ collagen/poloxamer sheet in type I diabetic mouse model

In Type I diabetes, a significant portion of beta cells are destroyed, leading to an insufficient secretion of insulin and increased wound healing time [9]. Thus, a type 1 diabetes mellitus was used in the current study to evaluate the effect
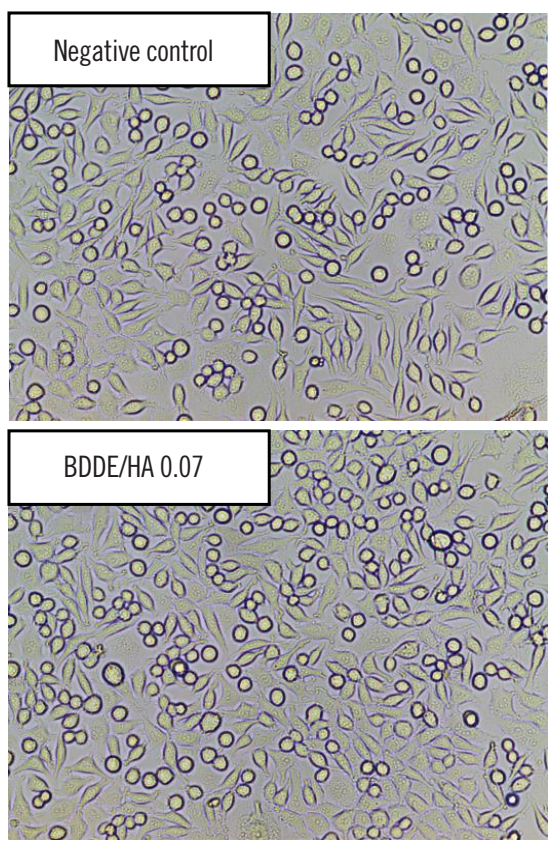
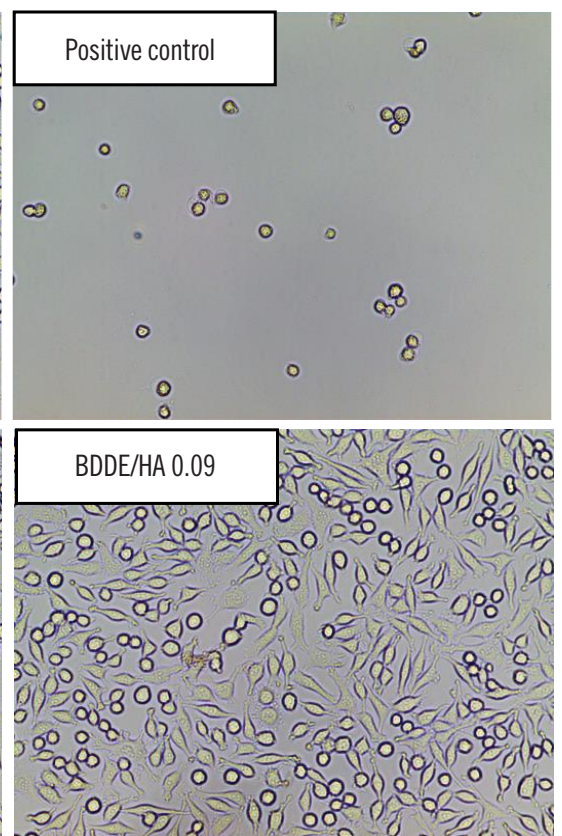

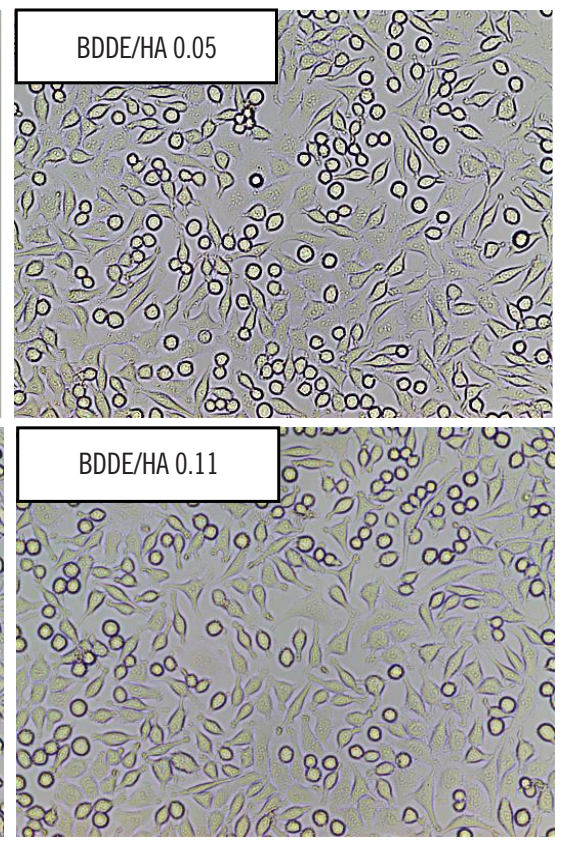

Fig. 6. Cell morphology and cytotoxicity test results of C-HA/collagen/poloxamer Sheet.
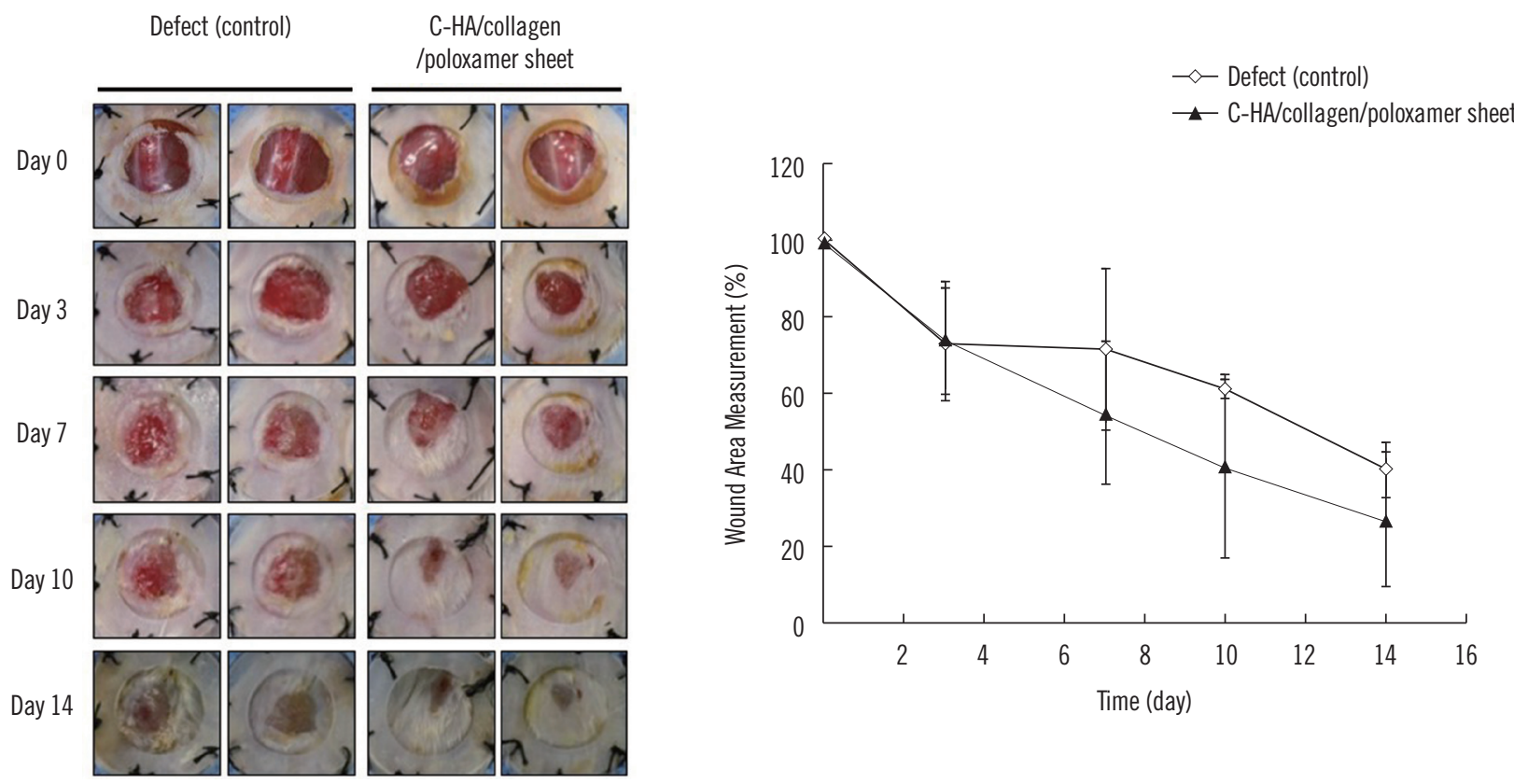

Fig. 7. Wound recovery results. 
of the C-HA/collagen/poloxamer sheets on wound healing. Type I diabetes was induced by STZ. Blood glucose levels were measured prior to the experiment to verify that they were $\geq 300 \mathrm{mg} / \mathrm{dL}$.

A minimally absorption sheet, made with a BDDE/HA molar ratio of 0.11 , was selected to assess whether or not the sheets would have adequate absorption when used for the treatment of chronic wounds and in wound environments characterized by excessive exudate.

In order to examine the effect of the sheets under diabetic conditions, the mice were sacrificed on days 7 and 14 after surgery. The wound area was photographed on the day of surgery and on days 3, 7, 10, and 14 postoperatively. It was shown that the wound area decreased over time (Fig. 7).

The C-HA/collagen/poloxamer product completely absorbed the exudate in the early stages of wound recovery when the amount of exudate is the greatest. Measurement of the wound areas revealed that wound healing in the group treated with the $\mathrm{C}-\mathrm{HA} /$ collagen/poloxamer sheets accelerated from the third postoperative day, with most of the wounds healing by day 14 , compared to those in the control (defect) group. Following H\&E staining, it was shown that the experimental group achieved $40 \%$ and $80 \%$ re-epithelialization by days 7 and 14 postoperatively, respectively, compared to reepithelialization of $60 \%$ in the control group on the 14th day. The results demonstrated that use of the product improved wound healing rate (Fig. 8).

The alignment and formation of collagen fibers was assessed to determine the degree of scarring during the wound healing process. MT staining was performed to observe and quantify newly formed collagen fibers at the wound site. Excellent alignment and adhesion of the collagen fibers was demonstrated with the use of the C-HA/collagen/poloxamer sheets. Quantitative analysis was also performed to measure the newly formed collagen fibers at the wound site using Image J software. A collagen deposition ratio of $39 \% \pm 17 \%$ was
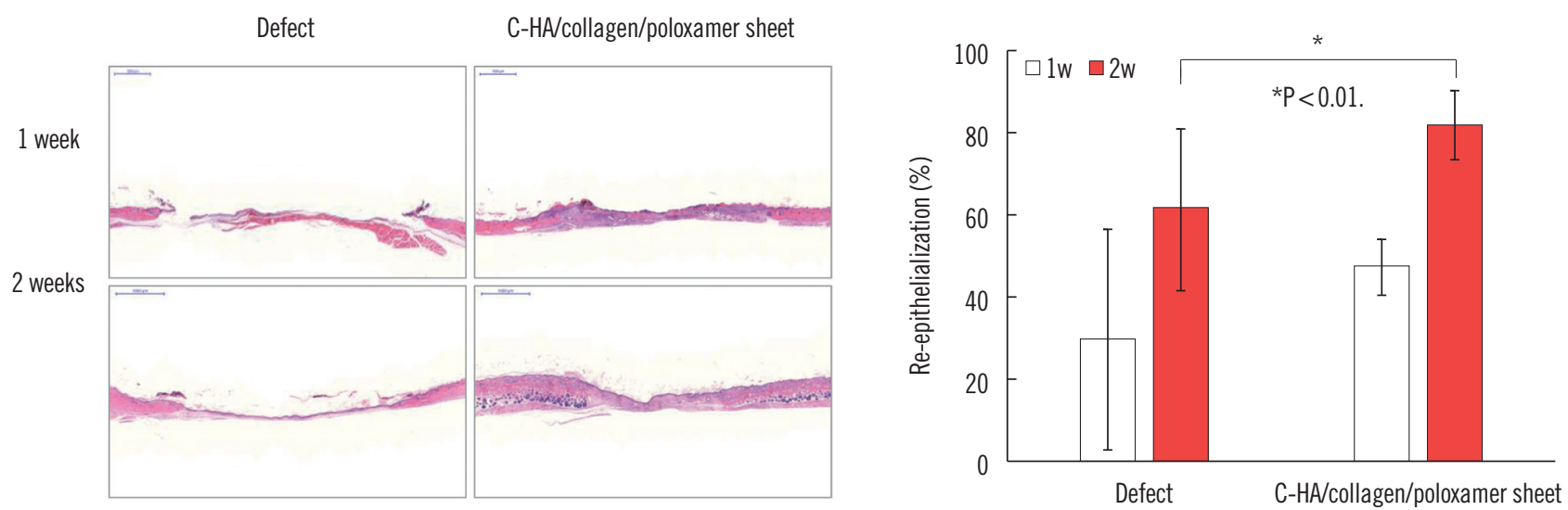

Fig. 8. Re-epithelialization of C-HA/collagen/poloxamer sheet (H\&E stain, $\times 40)$.
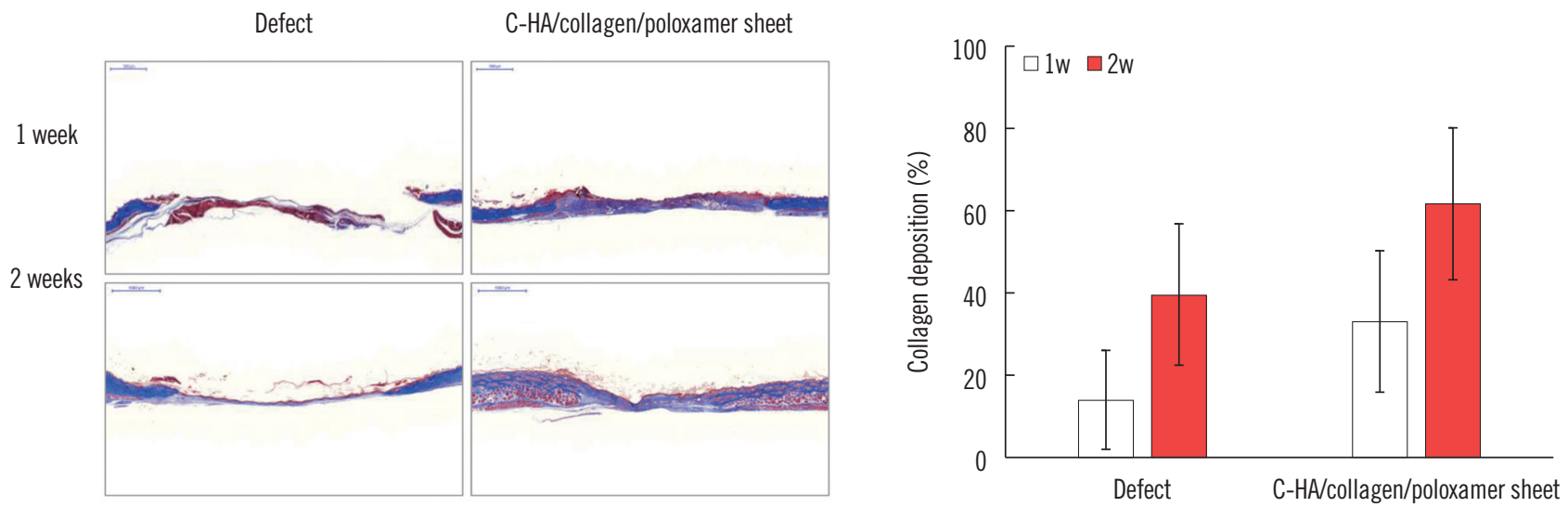

Fig. 9. Collagen deposition of C-HA/collagen/poloxamer Sheet (MT stain, $\times 40$ ). 
recorded in the control group, compared with a comparative figure of $61 \% \pm 18 \%$ in the experimental group after a twoweek period, thus confirming that the sheet can induce collagen alignment and deposition (Fig. 9).

\section{Discussion}

Combining the use of collagen and other materials is increasingly being performed to compensate for the limitations of $\mathrm{HA}$ [10-12]. Studies on the preparation and properties of HA, and on its use in various applications in the wound dressing market, are actively underway $[11,13]$. The focus in recent years has been on the replacement of or an improvement in $\mathrm{HA}$ and collagen products in the wound dressing market to ensure greater ease of use. Since numerous research findings are available in the literature on the combined use of HA and collagen [10,12], a decision was made in the current study to manufacture a C-HA and collagen composite sheet with porous support to facilitate wound healing. This study aimed to fabricate a C-HA/collagen composite sheet as a porous support to facilitate wound healing.

This study also aimed to improve the drawbacks of C-HA sheets developed by previous studies, such that it was necessary to carry out the whole cross-linking process in the form of a sheet to produce a C-HA sheet and to perform a long purification process to remove the cross-linking agent according to the thickness of the film during the purification process and that insufficient purification process may impose a risk of toxicity by residual cross-linking agents. A key study criterion was to produce a sheet that could withstand the conditions necessary to shorten the manufacturing process, while simultaneously expediting wound healing.

Instead of producing a cross-linked product initially in sheet form, the C-HA product was first prepared for use as a sheet with cross-linking properties so that it could be manufactured easily. The C-HA mass obtained through a crosslinking reaction was cut to a certain size and purified to the extent that there was no residual cross-linking agent, and this process was completed within 24 hours. It was established that the sheets, manufactured by mixing the purified and pulverized C-HA product with collagen and poloxamer, followed by freeze-drying, had a uniform porous structure.

It was expected that the sheet properties would change according to the BDDE/HA molar ratios. However, the tensile strength and surface structure of the sheets were not significantly affected by this, nor by the HA, collagen, and polox- amer ratios. Likewise, the BDDE/HA molar ratio did not affect tensile strength, even after moisture absorption. It is likely that the product remained affected, regardless of the BDDE/ HA molar ratio, since it was produced after the C-HA product was manufactured and pulverized, meaning that it was not cross-linked as a whole. Similar to the findings of other studies $[14,15]$, it was demonstrated in the current research that the sheet strength decreased after the sheets were wet.

It was observed that the higher the C-HA molar ratio and the lower the degree of cross-linking, the greater the absorptivity of the sheets. Little difference in absorptivity was noted within a range of cross-linking ratios. Theoretically, the lower the degree of cross-linking, the higher rate of absorption [16, 17], suggesting that there is a critical point at which the increase in absorption by cross-linking significantly exceeds the intrinsic absorptive property of the raw material. If stronger properties are desired for a sheet being produced, the solid content of the sheet manufacturing solution should be increased, but it is likely that such a sheet would be too rigid (owing to difficulties with application over a curved region) for use as a wound dressing. Therefore, the manufacture of this type of sheet was not pursued in the present study.

Since the primary application of the sheet created was for use as a dressing material for chronic wounds, the objective was to maintain its shape without its disintegration before the next dressing replacement. A dressing replacement cycle is usually $2-3$ days (or seven days at most), thus the potential for disintegration following immersion of the dressing material in PBS over a seven-day period was studied. Although the product manufactured in this study was expected to be at risk of disintegrating in a wet environment, almost no change in appearance and only a small change in weight was observed when it was placed in PBS at $37^{\circ} \mathrm{C}$ for seven days, possibly because it had not been cross-linked as a whole. It is believed that that the dressing retained its shape because it was physically entangled between molecular chains during the pulverization process of the C-HA.

To evaluate the safety, a cytotoxicity test was performed according to the BDDE/HA molar ratio, and it was confirmed that no toxicity was observed in all groups. Considering that $\mathrm{HA}$ and collagen are materials that are typically used to facilitate wound healing, the porous structure and biocompatibility of the sheets could be used to support the reconstitution of biological tissue.

In the current study, the efficacy of this type of sheet in wound regeneration was evaluated in a chronic wound 
model, in which the wound healing process is characterized by delays and a high exudate environment. Therefore, effective exudate absorption is a key selection factor when considering a dressing material. For this reason, the lowest absorbent sheet (with a BDDE/HA molar ratio of 0.11) was selected because if its wound healing properties could be demonstrated, then it could also be assumed that the other $\mathrm{C}-\mathrm{HA} /$ collagen/poloxamer sheets would have adequate absorbency for use in the treatment of chronic wounds. When the C-HA/ collagen/poloxamer sheets were applied to wounds of a similar size in the type 1 diabetes mellitus chronic wound model, the wounds were recovered $50 \%$ by day 7 (based solely on a visual inspection), while the re-epithelialization and collagen deposition ratios were empirically established to be $60 \%$ and $40 \%$, respectively, using H\&E staining. Thus, more rapid recovery was seen in the group treated with the product that that achieved in the untreated group. On day 14 , re-epithelialization of $80 \%$ was recorded, i.e., almost complete recovery, and the collagen deposition ratio was roughly twice that of the untreated group. These results suggest that the C-HA/ collagen/poloxamer sheets manufactured in the current study facilitated wound healing.

\section{Conclusions}

An easily manufactured $\mathrm{C}-\mathrm{HA} /$ collagen/poloxamer sheet was created in the current study, and its characteristics were assessed in terms of characteristics, safety, viscosity, appearance, morphology, tensile strength, absorption, and disintegration. The product, with physical properties that are suitable for application to wounds, was confirmed to be safe in the treatment thereof. Given that wound healing was enhanced when used in a type 1 diabetes mellitus model, the C-HA/collagen/poloxamer sheets manufactured in this study have the potential to be used as a dressing for chronic wounds.

\section{Conflict of interest}

No potential conflict of interest relevant to this article was reported.

\section{Acknowledgements}

This research was supported by Creative Materials Discovery Program through the National Research Foundation of Korea (NRF) funded by the Ministry of Science, ICT and Future
Planning (NRF-2016M3D1A1026533).

\section{References}

1. Kong MK, Lee PC. Evaluation of a pretreatment method for two-dimensional gel electrophoresis of synovial fluid using cartilage oligomeric matrix protein as a marker. J Microbiol Biotechnol 2012;22(5):654-8.

2. Simoni RD, Hill RL, Vaughan M, et al. The discovery of hyaluronan by Karl Meyer. J Biol Chem 2002;277(39):e27.

3. Chen WY, Abatangelo G. Functions of hyaluronan in wound repair. Wound Repair Regen 1999;7(2):79-89.

4. Laurent TC, Fraser JR. Hyaluronan. FASEB J 1992;6(7): 2397-404.

5. Lee M, Han SH, Choi WJ, et al. Hyaluronic acid dressing (Healoderm) in the treatment of diabetic foot ulcer: A prospective, randomized, placebo-controlled, single-center study. Wound Repair Regen 2016;24(3):581-8.

6. Price RD, Berry MG, Navsaria HA. Hyaluronic acid: the scientific and clinical evidence. J Plast Reconstr Aesthet Surg 2007;60(10):1110-9.

7. De Boulle K, Glogau R, Kono T, et al. A review of the metabolism of 1,4-butanediol diglycidyl ether-crosslinked hyaluronic acid dermal fillers. Dermatol Surg 2013;39(12): 1758-66.

8. Park SN, Lee HJ, Lee KH, et al. Biological characterization of EDC-crosslinked collagen-hyaluronic acid matrix in dermal tissue restoration. Biomaterials 2003;24(9):1631-41.

9. Lima MH, Caricilli AM, de Abreu LL, et al. Topical insulin accelerates wound healing in diabetes by enhancing the AKT and ERK pathways: a double-blind placebo-controlled clinical trial. PLoS One 2012;7(5):e36974.

10. Kim ZH, Lee Y, Kim SM, et al. A composite dermal filler comprising cross-linked hyaluronic acid and human collagen for tissue reconstruction. J Microbiol Biotechnol 2015; 25(3):399-406.

11. Anilkumar TV, Muhamed J, Jose A, et al. Advantages of hyaluronic acid as a component of fibrin sheet for care of acute wound. Biologicals 2011;39(2):81-8.

12. Niiyama $H$, Kuroyanagi Y. Development of novel wound dressing composed of hyaluronic acid and collagen sponge containing epidermal growth factor and vitamin $\mathrm{C}$ derivative. J Artif Organs 2014;17(1):81-7.

13. Park HS, Kim AR, Noh I. Physical and Biological Evaluations of Cross-linked Hyaluronic Acid Film. Biomater Res 2013; 17(4):153-9. 
Kang $\mathrm{HJ}$ et al.

Wound healing of cross-linked sheets

14. Ashl ey RJ Permeability and plastics packaging. Polymer Pernreabilily. 1985;269-308. London:Elsevier Applied Science Publishers, Ltd.

15. Gennadios A, Park HJ, Weller CL. Relative Humidity and Temperature Effects on Tensile Strength of Edible Protein and Cellulose Ether Films. Trans ASAE 1993;36(6):1867-72.
16. Lee DY, Cheon C, Son S, et al. Influence of molecular weight on swelling and elastic behavior of hyaluronic acid dermal fillers. Polymer Korea 2015;39:976-80.

17. Kim J, Choi J, Lee DY. Pyrogenicity of hyaluronic acid hydrogel crosslinked by divinyl sulfone for soft tissue augmentation. Nat Sci 2010;2:764-8. 